\title{
Conhecimento de estudantes portugueses de enfermagem sobre doação de sangue
}

Knowledge of Portuguese nursing students about blood donation

Conocimientos de estudiantes portugueses de Enfermería sobre donación de sangre

Lorena Casal-Otero ${ }^{1}$ id https://orcid.org/0000-0002-0906-4321

Ermelinda Marques² ${ }^{2}$ iD https://orcid.org/0000-0002-8246-0101

Alba-Elena Martínez-Santos ${ }^{1}$ id https://orcid.org/0000-0002-9051-9185

Raquel Rodríguez-González¹ io https://orcid.org/0000-0003-4171-0263

Josefa del Carmen Fernández-de-la-Iglesia ${ }^{1}$ ic https://orcid.org/0000-0002-8320-7239

\section{Como citar:}

Casal-Otero L, Marques E, Martínez-Santos A-E, Rodríguez-González R, Fernández-de-la-Iglesia

JC. Conhecimento de estudantes portugueses de enfermagem sobre doação de sangue. Acta Paul Enferm. 2020;33:APE20190166.

DOI http://dx.doi.org/10.37689/actaape/2020A00166

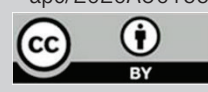

Descritores

Doadores de sangue: Estudantes de enfermagem; Educação superior; Conhecimentos, Atitudes e prática em saúde

Keywords

Blood donors; Students, nursing: Education, higher; Health knowledge, attitudes, practice

Descriptores

Donantes de sangre; Estudiantes de enfermeira; Educación Superior; Conocimientos, actitudes y

práctica en salud

Submetido 9 de Julho de 2019

Aceito

7 de Outubro de 2019

Autor correspondente Josefa del Carmen Fernández de la Iglesia c.delaiglesia@usc.es

\section{Resumo}

Objetivo: Avaliar o nível de conhecimento de estudantes de Enfermagem face à doação de sangue; identificar a importância atribuída às barreiras para a doação de sangue; e, identificar os meios de comunicação digital mais utilizados.

Métodos: Trata-se de uma pesquisa realizada numa Instituição de Ensino Superior da Região Centro de Portugal no ano letivo de 2018/2019. 0 estudo é descritivo, de natureza transversal, através de dois questionários ad hoc, preenchido on-line por 165 estudantes de Enfermagem.

Resultados: Os conhecimentos podem ser considerados de muito baixos (intervalo entre as doações; tempo de espera depois de fazer uma tatuagem ou um piercing; tempo de espera entre a ingestão de ferro; baixos (quantidade de sangue e alimentação prévia), médios (idade e peso mínimo, dador universal) e altos (casos em que o sangue é analisado). Os motivos para não doar sangue que assumiram bastante e muita importância foram "Porque tenho alguma condição física ou médica que me impede doar" e "Porque mantive práticas sexuais de risco".

Conclusão: A falta de conhecimentos sobre as condições de elegibilidade pode fazer com que uma pessoa considere, de forma errada, que não pode doar sangue. Tendo em conta os resultados obtidos e o acesso aos meios de comunicação digital, sugere-se que sejam implementados programas de e-saúde que promovam 0 aumento de conhecimentos e a redução de barreiras à doação de sangue.

\section{Abstract}

Objective: To assess the level of knowledge of nursing students regarding blood donation, to identify the importance consider challenges for blood donation, and to determine the most used digital communication channels.

Methods: This research was performed at University of Central Portugal in 2018/2019. This was a descriptive cross-sectional study. A total of 165 nursing students completed online two ad hoc questionnaires.

Results: The level of knowledge can be classified as very low (interval between donations, waiting time after getting a tattoo or piercing, waiting time after taking iron supplements), low (amount of blood, eating before donation), medium (age and minimum weight, universal donor) and high (cases in which the blood was analyzed). The reasons for not donating blood that gained considerable and great importance were: "Because I have a physical or medical condition that makes me ineligible to donate" and "Because I had a risky sexual behavior". 
Conclusion: The lack of knowledge about the eligibility requirements can make someone wrongly consider that he or she cannot donate blood. Considering the results obtained and the access to digital communication channels, the implementation of eHealth programs is recommended to promote more knowledge, and to reduce the barriers for blood donation.

\section{Resumen}

Objetivo: Evaluar el nivel de conocimiento de estudiantes de Enfermería sobre la donación de sangre, identificar la importancia atribuida a las barreras para la donación de sangre e identificar los medios de comunicación digital más utilizados.

Métodos: Se trata de un estudio realizado en una Institución de Enseñanza Superior de la región centro de Portugal en el año lectivo 2018/2019. El estudio es descriptivo, de naturaleza transversal, a través de dos cuestionarios ad hoc que fueron completados en línea por 165 estudiantes de Enfermería.

Resultados: Los conocimientos pueden considerarse muy bajos (intervalo entre las donaciones, tiempo de espera después de hacerse un tatuaje o piercing, tiempo de espera después de la ingesta de hierro); bajos (cantidad de sangre y alimentación previa); medios (edad y peso mínimo, donante universal) y altos (casos en que se analiza la sangre). Los motivos para no donar sangre que tuvieron bastante y mucha importancia fueron "Porque tengo alguna condición física o médica que me impide donar" y "Porque mantuve prácticas sexuales de riesgo".

Conclusión: La falta de conocimientos sobre las condiciones de elegibilidad puede provocar que una persona considere, de forma equivocada, que no puede donar sangre. Teniendo en cuenta los resultados obtenidos y el acceso a los medios de comunicación, se sugiere implementar programas de eSalud que promuevan el aumento de conocimientos y la reducción de barreras para la donación de sangre.

\section{Introdução}

O sangue é um elemento essencial para a vida que não pode ser substituído artificialmente. A doação de sangue voluntária, responsável e altruísta é o único meio para cobrir as crescentes necessidades decorrentes do aumento da esperança de vida e dos procedimentos médico-cirúrgicos. Assim, recrutar novos doadores ou fidelizar aqueles que já doaram é um objetivo fundamental para que haja doaçôes suficientes e que garantam a autossuficiência das transfusóes, necessárias diariamente.

O Sistema Português de Hemovigilância está implementado em Portugal desde 2008 dando cumprimento ao Decreto-Lei 267/2007 de 24 de julho, o qual transpôs para a ordem jurídica interna as Diretivas Europeias 2002/98/EC do Parlamento Europeu e do Conselho e a Diretiva 2005/61/EC da Comissão Europeia. A responsabilidade de assegurar o funcionamento do Sistema Português de Hemovigilância é atribuída ao Instituto Português do Sangue e da Transplantação (IPST). ${ }^{(1)}$

A doação de sangue pode fazer-se nos Centros de Sangue e Transplantação de Lisboa, Porto e Coimbra ou serviços hospitalares com recolha de sangue. $\mathrm{O}$ planejamento das sessóes de coleta por dia, distrito, distrito e conselho, local e centro de coleta está disponível na web do IPST. O potencial doador deve apresentar um documento de identificação com fotografia e preencher um questionário. Em seguida é avaliado por um profissional de saúde qualificado que determina a sua elegibilidade para a doação de sangue, através de uma avaliação clínica e exame físico. O Cartão Nacional de Dador de Sangue identifica o dador de sangue e contém os registos das doaçôes efetuadas constantes na base de dados do cartão nacional de doador. ${ }^{(2)}$

Em Portugal, assistimos nos últimos anos a um decréscimo significativo no número de novos doadores e no número de unidades de sangue obtidas. ${ }^{(3)}$ Estudos recentes revelam que em Portugal nos últimos 7 anos o número de doadores diminuiu em $40 \%{ }^{(4)}$

Nesse contexto, captar e fidelizar doadores em instituições de ensino superior torna-se um fato crucial. Os jovens são um grupo ideal porque têm muitos anos pela frente para poderem doar, além disso, devido às suas características e dinamismo, os jovens tendem a ser saudáveis, idealistas e motivados, constituindo assim, um excelente grupo de potenciais doadores voluntários de sangue não remunerado. ${ }^{(5-7)}$

Promover a doação de sangue entre o grupo de estudantes do ensino superior implica a necessidade de investigar os fatores que podem promovê-la ou inibí-la. Para isso é necessário conhecer quais os elementos que estão associados à doação ou se as pessoas possuem os conhecimentos adequados para tomar a decisão de doar ou não doar sangue. ${ }^{(8)}$

Relativamente aos conhecimentos, diferentes estudos referem que os conhecimentos sobre a doação de sangue são insuficientes e que muitos jovens detém ideias erradas, ${ }^{(9)}$ sobre as quais se deve trabalhar. ${ }^{(7)} \mathrm{A}$ investigação neste campo é crucial por dois motivos. Em primeiro lugar constata-se que existe uma maior motivação face à doação quando as pessoas estão bem 
informadas sobre o processo e os benefícios. ${ }^{(10)} \mathrm{e}$, em segundo lugar, esta informação permitirá aos responsáveis das políticas de promoção da saúde, desenhar campanhas formativas contextualizadas, e adaptadas a diferentes grupos etários ${ }^{(1)}$ que incrementem o conhecimento dos estudantes ${ }^{(12)}$ e que possam prever estratégias, mensagens e intervençóes ${ }^{(4)}$ que produzam impacto.

Por outro lado, a revisão da literatura ajudou-nos a identificar diferentes barreiras pelas quais os jovens decidem não doar sangue. Estes obstáculos fazem referência, em primeiro lugar, a razóes pessoais ou preconceitos, por exemplo, causas médicas, ${ }^{(4,6)}$ medo de contrair alguma doença ou infecçáa ${ }^{(9,12,13)}$ ou acreditar que se comercializa o sangue. ${ }^{(4,11)}$ Em segundo lugar, identificam-se uma série de barreiras relacionadas com medos, como o medo da dor ${ }^{(9,12,14)}$ no procedimento de extração/agulha ${ }^{(4,13,15)}$ ou rejeição ao ambiente hospitalar, ${ }^{(11,15)}$ entre outros. Identificaramse também diferentes obstáculos que podemos denominar pretextos, entre os quais, não pensar em doar, ${ }^{(4,14)}$ falta de tempo, ${ }^{(9,12,13)}$ que ninguém lhe pediu $^{(4,9,12)}$ e ter pouca informaçáo. ${ }^{(9,15)}$

Este estudo busca aportar evidências sobre conhecimentos e barreiras à doação de sangue em estudantes portugueses de ensino superior, especificamente futuras enfermeiras, uma vez que existem poucas evidências científicas sobre o assunto neste país onde a taxa de doação (32,4 por 1000 habitantes) segue distanciando-se das taxas de países vizinhos como a Espanha; ainda que esteja na margem dos países de alta renda segundo a OMS. ${ }^{(16-18)}$ Os resultados apresentados podem ser úteis para que os provedores de saúde (enfermeiras e outros) elaborem intervençóes para aumentar a doação de sangue em estudantes de ensino superior. Em Portugal, os jovens entre 18 e 24 anos mal chegam a 14,35\% do total de doadores, embora o IPST aposta na promoção, sensibilização e educação para a doação de sangue em Escolas Básicas e de Ensino Superior entre outras organizaçóes sociais. ${ }^{(16,19)}$

Face ao exposto e em consonância com os autores consultados, os objetivos do nosso estudo são:

1. Avaliar o nível de conhecimento face à doação de sangue que tem os estudantes de Enfermagem de uma instituição de ensino superior da região centro de Portugal;
2. Identificar a importância atribuída às barreiras pelos estudantes para a doação de sangue.

3. Identificar os meios de comunicação digital mais utilizados pelos estudantes de Enfermagem e que possam ser úteis para as campanhas de recrutamento.

\section{Métodos}

Trata-se de um estudo descritivo, transversal, com abordagem quantitativa, no qual participaram 165 estudantes do Curso de Graduação em Enfermagem (do primeiro ao quarto ano) de uma instituição de ensino superior da região centro de Portugal. A coleta de dados ocorreu no ano letivo 2018/2019. Os alunos que participaram das aulas nos meses de janeiro e fevereiro de 2019 foram convidados a participar e os questionários ad hoc puderam ser preenchidos on-line durante esse período. A natureza e o propósito da investigação foram explicados juntamente com as informaçôes de contato do investigador; bem como se obteve uma declaração de consentimento de uso de dados, enfatizando que eram anônimos.

Para medir os conhecimentos e as atitudes sobre a doação de sangue utilizaram-se dois questionários. O primeiro foi elaborado para medir o nível de conhecimento e consistia em 10 perguntas, com várias opçóes de resposta, em que apenas uma das opçóes era a resposta correta (obtendo uma pontuação entre 0 e 10 pontos). As variáveis dependentes do estudo foram as respostas corretas sendo a avaliação do nível de conhecimento sobre a doação de sangue obtida a partir das 10 questóes do questionário ad hoc.

Da mesma forma, construiu-se outro questionário para identificar a importância atribuída às barreiras face à doação de sangue, no sentido de conhecer os motivos que justificavam não doar sangue, através de uma escala Likert de 0 a 5 pontos na qual os participantes estabeleceram o grau de importância (desde nada importante a muito importante), agrupando-se em 3 dimensóes: razóes pessoais e preconceitos, medos e pretextos (Quadro 1). Estudos anteriores e as normas do IPST foram levados em conta. A análise de fiabilidade da escala das barreiras 
face à doação de sangue mostrou um índice de alfa de Crohnbach de 0.893, indicando assim uma alta consistência interna.

Quadro 1. Barreiras face à doação de sangue

\begin{tabular}{|c|c|c|}
\hline \multirow{9}{*}{ 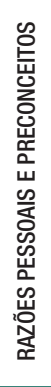 } & 11 & Porque mantive práticas sexuais de risco \\
\hline & 12 & Porque fiz uma tatuagem, piercing ou acupuntura \\
\hline & 13 & Porque tenho alguma condição física ou médica que me impede doar \\
\hline & 14 & Por causa das minhas crenças religiosas \\
\hline & 15 & Porque tenho medo de ficar doente \\
\hline & 16 & Porque desconfio da esterilidade do material \\
\hline & 17 & $\begin{array}{l}\text { Porque os meus pais, amigos ou familiares me disseram para não } \\
\text { doar sangue }\end{array}$ \\
\hline & 18 & Porque acho que o sangue é comercializado \\
\hline & 19 & $\begin{array}{l}\text { Porque estou desconfortável com as perguntas da entrevista antes } \\
\text { da doação }\end{array}$ \\
\hline \multirow{5}{*}{$\begin{array}{l}\text { 号 } \\
\stackrel{\mathrm{m}}{\Sigma}\end{array}$} & II1 & Por medo da dor \\
\hline & $\| 12$ & Porque tenho medo do procedimento de doação de sangue \\
\hline & $\| 13$ & Porque temo a perda de sangue \\
\hline & $\| 4$ & Porque tenho medo do ambiente hospitalar \\
\hline & 1115 & Porque doar enfraquece o meu corpo \\
\hline \multirow{5}{*}{ 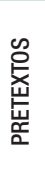 } & III1 & Porque não penso em doar sangue \\
\hline & III2 & Por falta de tempo \\
\hline & III3 & Porque não sei onde ir doar \\
\hline & III4 & Porque ninguém me pediu \\
\hline & III5 & Porque tenho pouca informação sobre a doação \\
\hline
\end{tabular}

A análise estatística foi realizada no SPSS 20.0 para Mac (IBM inc.). Utilizaram-se análises descritivas (frequências, percentagens e médias) para descrever a amostra. Tendo em conta que a distribuição não era normal e as variáveis eram ordinais, utilizaram-se testes não paramétricos para comprovar se existiam diferenças significativas relativamente ao nível de conhecimento e às barreiras vivenciadas. Estabeleceu-se um nível de significância (alfa) em 0.05.

Para analisar se existiam diferenças relativamente ao nível de conhecimento e às barreiras, em função da variável gênero e ter sido doador previamente, utilizou-se o teste U de Mann-Whitney. Em todos os casos, foi primeiro realizada a comparação utilizando a média das pontuaçóes dos itens que compunham cada dimensão. Posteriormente, para os casos em que se obtiveram diferenças significativas, realizaram-se as comparaçóes para cada item dessas dimensóes.

A realização desta pesquisa foi autorizada pelo Diretor da Instituição e respeitou todos os princípios éticos inerentes à pesquisa com seres humanos. Obteve parecer favorável da Comissão de Ética da instituição de ensino (parecer n $5 / 2019$ ).

\section{Resultados}

Do total de participantes, $84.4 \%$ dos participantes eram mulheres, $13.9 \%$ eram homens e $1.2 \%$ eram transexuais. A média de idades foi de 21.25 anos (DT 3.07). Relativamente à doação de sangue, apenas $21.8 \%$ era dador, não sendo este dado explicado pela existência de problemas que impedissem a doação, uma vez que apenas $18,8 \%$ deu essa informação. Praticamente todos os alunos (98.2\%) consideraram necessária a doação de sangue.

Os resultados mostraram que o nível geral de conhecimentos da amostra avaliada no presente estudo é baixo ( $M=4.00$, $T D$ 1.71). Para realizar a análise individual de cada item avaliado, a pontuação foi obtida através da atribuição às frequências absolutas das respostas corretas, as percentagens divididas em quartis: de $0 \%$ a $25 \%$, de $26 \%$ a $50 \%$, de $51 \%$ a $75 \%$ e de $76 \%$ a $100 \%$; essas percentagens associam-se aos descritores "conhecimento muito baixo", "conhecimento baixo", "conhecimento médio" e "conhecimento alto", respectivamente. As questóes que indicam um menor nível de conhecimentos são as que se relacionam com a frequência com que se pode doar e com o tempo que deve passar após ter realizado uma tatuagem ou piercing, depois de ter tomado ferro ou o tempo que se deve esperar para beber álcool após a doação (Quadro 2).

Relativamente às barreiras para a doação de sangue, os participantes atribuíram maior importância às seguintes (Quadro 3): "ter alguma condição física ou médica que me impede de doar" $(\mathrm{M}=3.04)$, "manter práticas sexuais de risco" $(\mathrm{M}=2.36)$, "não saber onde doar" $(\mathrm{M}=2.27)$, "ter pouca informação sobre a doação" $(\mathrm{M}=2.16)$, "ter feito uma tatuagem, piercing ou acupunctura” $(\mathrm{M}=2.15)$.

Não foram encontradas diferenças estatisticamente significantes nas atitudes e nível de conhecimento dos participantes de acordo com a variável género. Apenas, foram encontradas diferenças estatisticamente significativas sobre o nível de conhecimento em função da variável já ter doador ( $\mathrm{U}=$ 1481.500,00, $\mathrm{p}=0.001$ ), sendo os participantes que tinham doado os que obtiveram pontuaçóes significativamente mais altas em conhecimentos sobre a 
doação de sangue. $\mathrm{Na}$ análise por itens, constatouse que as pessoas que já doaram apresentaram níveis de conhecimento significativamente mais altos sobre as questôes "quantidade de sangue que se extrai” ( $\mathrm{U}=1767.000, \mathrm{p}=0.011)$, "grupo sanguíneo

Quadro 2. Nível de conhecimento de estudantes de um Curso de Graduação em Enfermagem sobre a doação de sangue

\begin{tabular}{|c|c|c|c|}
\hline Questão & $\begin{array}{l}\text { Resposta } \\
\text { correta }\end{array}$ & $\begin{array}{l}\text { Proporção } \\
\text { (frequência) de } \\
\text { sucesso } \\
n(\%)\end{array}$ & $\begin{array}{c}\text { Nível de } \\
\text { conhecimento }\end{array}$ \\
\hline $\begin{array}{l}\text { Qual é a idade mínima para doar } \\
\text { sangue? }\end{array}$ & 18 anos & $108(65,5)$ & $\begin{array}{l}\text { conhecimento } \\
\text { médio }\end{array}$ \\
\hline $\begin{array}{l}\text { Qual é o peso corporal mínimo para } \\
\text { doar sangue? }\end{array}$ & $50 \mathrm{Kg}$ & $117(70,9)$ & $\begin{array}{l}\text { conhecimento } \\
\text { médio }\end{array}$ \\
\hline $\begin{array}{l}\text { Com que frequência se pode doar } \\
\text { sangue (intervalo mínimo entre } \\
\text { doações)? }\end{array}$ & 2 meses & $21(12,7)$ & $\begin{array}{l}\text { muito baixo } \\
\text { conhecimento }\end{array}$ \\
\hline $\begin{array}{l}\text { Quanto tempo é necessário esperar } \\
\text { para doar sangue depois de fazer } \\
\text { uma tatuagem ou um piercing }\end{array}$ & 4 meses & $23(13,9)$ & $\begin{array}{c}\text { muito baixo } \\
\text { conhecimento }\end{array}$ \\
\hline $\begin{array}{l}\text { Eu tive anemia e tomei ferro } \\
\text { oralmente. Quanto tempo é } \\
\text { necessário esperar entre a ingestão } \\
\text { de ferro e a doação? }\end{array}$ & 2 meses & $12(7,3)$ & $\begin{array}{l}\text { muito baixo } \\
\text { conhecimento }\end{array}$ \\
\hline $\begin{array}{l}\text { Que quantidade de sangue é } \\
\text { extraído numa doação? }\end{array}$ & $450 \mathrm{ml}$ & $70(42,4)$ & $\begin{array}{c}\text { baixo } \\
\text { conhecimento }\end{array}$ \\
\hline $\begin{array}{l}\text { Qual é o grupo sanguíneo } \\
\text { considerado doador universal? }\end{array}$ & $0-$ & $100(60,6)$ & $\begin{array}{l}\text { conhecimento } \\
\text { médio }\end{array}$ \\
\hline $\begin{array}{l}\text { Em que casos o sangue doado é } \\
\text { analisado? }\end{array}$ & Sempre & $139(84,2)$ & $\begin{array}{c}\text { alto } \\
\text { conhecimento }\end{array}$ \\
\hline Para doar sangue, recomenda-se & $\begin{array}{c}\text { Ter ingerido } \\
\text { qualquer } \\
\text { alimento nas } \\
\text { horas anteriores }\end{array}$ & $50(30,3)$ & $\begin{array}{c}\text { baixo } \\
\text { conhecimento }\end{array}$ \\
\hline $\begin{array}{l}\text { Quanto tempo tenho que esperar } \\
\text { para ingerir álcool depois da doação } \\
\text { de sangue? }\end{array}$ & $\begin{array}{l}\text { Devo esperar } \\
\text { pelo menos } 6 \\
\text { horas }\end{array}$ & $21(12,7)$ & $\begin{array}{l}\text { muito baixo } \\
\text { conhecimento }\end{array}$ \\
\hline
\end{tabular}

considerado universal" ( $\mathrm{U}=1894.500, \mathrm{p}=0.046)$ e "consumo alimentar recomendado antes do procedimento da doação" ( $U=1654.500, \mathrm{p}=0.001$ ). Quanto aos meios digitais mais utilizados, 67.3\% dos alunos acessam o Instagram e o Facebook diariamente através de seus smartphones $(35.2 \%)$ ou seus smartphones e computadores (52.7\%). Devese notar que apenas $1.2 \%$ dos participantes não usam nenhuma rede social diariamente (Instagram, Facebook ou Twitter).

\section{Discussão}

Com base nos resultados encontrados, podemos afirmar que este estudo fornece informações importantes sobre os condicionalismos que limitam os processos de doação de sangue num grupo de estudantes de Enfermagem do ensino superior português, onde a taxa de doações diminuiu nos últimos anos. ${ }^{(16)}$ Esta informação é útil no desenho de campanhas de sensibilização e de promoção da doação de sangue.

Os resultados obtidos no nosso estudo mostram uma baixa percentagem de doadores de sangue entre o grupo de estudantes do ensino superior (21.8\%), resultado semelhante ao obtido em investigaçóes an-

Quadro 3. Importância atribuída às barreiras de estudantes de um Curso de Graduação em Enfermagem face à doação de sangue

\begin{tabular}{|c|c|c|c|c|c|}
\hline \multirow[b]{2}{*}{ Questão/escala Likert } & \multicolumn{5}{|c|}{ Proporção (frequência) } \\
\hline & $\begin{array}{l}\text { Esse motivo não é } \\
\text { importante } \\
\mathrm{n}(\%)\end{array}$ & $\begin{array}{c}\text { Esse motivo é pouco } \\
\text { importante } \\
\mathrm{n}(\%)\end{array}$ & $\begin{array}{l}\text { Esse motivo tem } \\
\text { alguma importância } \\
\mathrm{n}(\%)\end{array}$ & $\begin{array}{c}\text { Esse motivo é } \\
\text { bastante importante } \\
\mathrm{n}(\%)\end{array}$ & $\begin{array}{c}\text { Esse motivo é muito } \\
\text { importante } \\
\mathrm{n}(\%)\end{array}$ \\
\hline Tenho alguma condição física ou médica que me impede de doar & $56(33,9)$ & $8(4,8)$ & $28(17,0)$ & $20(12,1)$ & $53(32,1)$ \\
\hline Mantive práticas sexuais de risco & $91(55,2)$ & $9(5,5)$ & $11(6,7)$ & $22(13,3)$ & $32(19,4)$ \\
\hline Não sei onde ir doar & $72(43,6)$ & $25(15,2)$ & $37(22,4)$ & $13(7,9)$ & $18(10,9)$ \\
\hline Fiz uma tatuagem, piercing ou acupuntura & $88(53,3)$ & $16(9,7)$ & $26(15,8)$ & 19(11,5) & $16(9,7)$ \\
\hline Desconfio da esterilidade do material & $93(56,4)$ & $30(18,2)$ & $26(15,8)$ & $5(3,0)$ & $11(6,7)$ \\
\hline Por causa das minhas crenças religiosas & $100(60,6)$ & $18(10,9)$ & $25(15,2)$ & $13(7,9)$ & $9(5,5)$ \\
\hline Tenho pouca informação sobre a doação & $72(43,6)$ & $27(16,4)$ & $42(25,5)$ & $15(9,1)$ & $9(5,5)$ \\
\hline Falta de tempo & $94(57,0)$ & $31(18,8)$ & $27(16,4)$ & $6(3,6)$ & $7(4,2)$ \\
\hline Temo a perda de sangue & $98(59,4)$ & $29(17,6)$ & $30(18,2)$ & $2(1,2)$ & $6(3,6)$ \\
\hline Ninguém me pediu & $129(78,2)$ & $14(8,5)$ & $14(8,5)$ & $3(1,8)$ & $5(3,0)$ \\
\hline Não penso em doar sangue & $91(55,2)$ & $29(17,6)$ & $33(20,0)$ & $7(4,2)$ & $5(3,0)$ \\
\hline Tenho medo do procedimento da doação de sangue & $75(45,5)$ & $36(21,8)$ & $36(21,8)$ & $13(7,9)$ & $5(3,0)$ \\
\hline Tenho medo de ficar doente & 104(63,0) & $34(20,6)$ & $20(12,1)$ & $2(1,2)$ & $5(3,0)$ \\
\hline Por medo da dor & $78(47,3)$ & $40(24,2)$ & $33(20,0)$ & $10(6,1)$ & $4(2.4)$ \\
\hline Doar enfraquece o meu corpo & $87(52,7)$ & $47(28,5)$ & $22(13,3)$ & $5(3,0)$ & $4(2.4)$ \\
\hline Acho que 0 sangue é comercializado & $107(64,8)$ & $31(18,8)$ & $20(12,1)$ & $3(1,8)$ & $4(2,4)$ \\
\hline Tenho medo do ambiente hospitalar & $107(64,8)$ & $43(26,1)$ & $10(6,1)$ & $2(1,2)$ & $3(1,8)$ \\
\hline Os meus pais, amigos ou familiares me disseram para não doar sangue & $125(75,8)$ & $21(12,7)$ & 12(7.3) & $4(2.4)$ & $3(1,8)$ \\
\hline Estou desconfortável com as perguntas da entrevista antes da doação & $99(60,0)$ & $42(25,5)$ & 19(11.5) & $2(1.2)$ & $3(1,8)$ \\
\hline
\end{tabular}


teriores. ${ }^{(9,20)} \mathrm{A}$ avaliação do nível de conhecimento é importante porque os baixos conhecimentos afetam negativamente a taxa de doação de sangue. ${ }^{(9,21,22)} \mathrm{Os}$ resultados desta investigação mostraram que o nível de conhecimentos em relação à doação de sangue é baixo e os participantes dadores têm um grau de conhecimento maior do que os náo doadores. Um resultado semelhante foi encontrado em outros estudos. ${ }^{(9,11,12,23)}$ No entanto, os nossos dados diferem dos resultados obtidos em outros estudos, nos quais o nível de conhecimento dos estudantes foi considerado alto. ${ }^{(24-26)}$

Os participantes do estudo serão profissionais de saúde em pouco tempo, entáo a falta de conhecimento sobre doação de sangue é especialmente preocupante. É importante que eles estejam cientes da importância desta questão, uma vez que não só serão possíveis doadores, mas também responsáveis por promover e realizar açóes educativas na população. Os estudantes que participaram neste estudo atribuíram maior importância às barreiras relacionadas com "razóes pessoais e preconceitos" e com "pretextos". Neste sentido, o obstáculo "ter alguma condição física ou médica que me impede doar" foi aquele que os estudantes manifestaram com maior frequência, corroborando os dados obtidos em outras investigaçôes já realizadas neste âmbito, ${ }^{(14,15,20)}$ mas diferindo de outras realizadas em contexto português. $(4,27)$ "Ter mantido práticas sexuais de risco" e "ter feito uma tatuagem, piercing ou acupuntura" foram barreiras frequentemente identificados pelos participantes não explicitamente avaliadas em nenhuma das pesquisas referenciadas anteriormente.

Outros impedimentos apontados na nossa investigação relativamente à dimensão pretextos são" não sei onde doar "seguidos de" ter pouca informação sobre a doação", impedimentos que não aparecem em outros estudos similares nos quais as razóes mais referenciadas nesta dimensão, pretextos, são: "falta de tempo", ${ }^{(8,13,15)}$ e "porque ninguém me pediu". $(4,8,9,12)$

O fato de as razões para a dimensão "medos" não serem as mais indicadas pelos participantes, não deixou de nos surpreender, uma vez que esta é a barreira mais comum na maioria das investigações realizadas. ${ }^{(3,6,28)}$ Este resultado pode estar relaciona- do ao perfil acadêmico dos participantes do estudo, estudantes da Graduação em Enfermagem.

Um último aspeto investigado no nosso estudo está relacionado com a utilização dos meios de comunicação digital pelos estudantes do ensino superior, verificando-se que praticamente todos os participantes acessam diariamente as redes sociais. Este dado é importante porque nos informa sobre as redes que são utilizadas com maior frequência pelos estudantes, no sentido de se poderem canalizar possíveis campanhas de educação para a saúde e sensibilização para este grupo específico. Atualmente o IPST desenvolve atividades de promoção e educação através de oficinas e atividades com redes de televisão e filmes, bem como visitas a escolas, feiras e festas; podendo doar em sessões móveis ou num posto fixo. As estratégias através de redes sociais são pouco promovidas e devem ser estudadas e tidas em conta, uma vez que os estudantes portugueses percebem que podem ser úteis para adquirir conhecimento, expressar e publicar as suas próprias opiniōes. ${ }^{(16,19,29)}$

\section{Conclusão}

Este estudo foi realizado para avaliar o nível de conhecimento dos estudantes do ensino superior relativamente à doação de sangue, assim como para identificar a importância atribuída às barreiras relacionadas com a ação de doar. Pudemos constatar que o conhecimento sobre a doação de sangue é baixo pelo que é necessário incrementá-lo já que poderia ter influência nas taxas de doação; também identificamos obstáculos importantes que influenciam a decisão de doar. Procuramos também identificar os meios de comunicação digital mais utilizados pelos estudantes. Os estudantes de Enfermagem são um grupo específico, o qual podemos sensibilizar e conscientizar para a doação de sangue através de redes sociais, especialmente Instagram e Facebook. É necessário continuar investigando a influência do uso de redes sociais em campanhas de promoção de doação de sangue, bem como a melhoria na articulação de sinergias entre o sistema de saúde e instituiçôes de ensino para melhorar as taxas de doação entre os mais novos. 


\section{Colaborações}

Casal-Otero L, Marques E, Martínez-Santos A-E, Rodríguez-González R e Fernández-de-la-Iglesia JC contribuíram com a conceção do projeto, análise e interpretação dos dados, redação do artigo, revisão crítica relevante do conteúdo intelectual e aprovação final da versão a ser publicada.

\section{Referencias}

1. Instituto Português do Sangue e da Transplantação. Sistema Português de Hemovigilância [Internet]. Lisbon: Instituto Português do Sangue e da Transplantação; 2017 [citado 2019 Ago 28]. Disponível em: http:// ipst.pt/index.php/sistema-portugues-de-hemovigilancia

2. Serviço Nacional de Saúde (SNS). Doação de Sangue [Internet]. Temas da saúde: doação de sangue. Lisbon:SNS; 2019 [citado 2019 Ago 28]. Disponível em: https://www.sns24.gov.pt/tema/dadiva-e-transplante/ doacao-de-sangue/\#sec-0

3. Lemos B, Ferreira C, Zuzarte N, Nunes L. [Factors influencing blood donation, systematic literature review] RIASE. 2018;4(2):1443-59. Portuguese.

4. Gomes MJ, Nogueira AJ, Antão C, Teixeira C. Motivations and attitudes towards the act of blood donation among undergraduate health science students. Transfus Apheresis Sci. 2019;58(2):147-51.

5. World Health Organization (WHO). Club 25: Reaching young blood donors [Internet]. Genève: WHO; 2006 [cited 2019 Jun 26]. Avaliable from: http://www.who.int/worldblooddonorday/campaignkit/WBDD_ Club25_English.pdf?ua=1

6. Zito E, Alfieri S, Marconi M, Saturni V, Cremonesi G. Adolescents and blood donation: motivations, hurdles and possible recruitment strategies. Blood Transfus. 2012;10(1):45-58.

7. Özgür S, Ürek H, Kösal K. Turkish university students' opinions towards blood donation. Univ J Educ Res. 2018;6(5):897-908.

8. Raghuwanshi B, Pehlajani NK, Sinha MK. Voluntary blood donation among students - a cross-sectional study on knowledge and practice vs. attitude. J Clin Diagn Res. 2016;10(10):EC18-22.

9. Baig M, Habib H, H Haji A, T Alsharief F, M Noor A, G Makki R. Knowledge, misconceptions and motivations towards blood donation among university students in Saudi Arabia. Pak J Med Sci. 2013;29(6):1295-9.

10. World Health organization (WHO); International Federation of Red Cross. Towards 100 \% Voluntary Blood Donation: A global framework for Action [Internet]. Genève: WHO; 2010. [cited 2019 Jul 29]. Avaliable from: http://www.who.int/iris/handle/10665/44359

11. Alfieri S. Representations and motivations of blood donation in adolescence through a mixed method approach. Transfus Apheresis Sci. 2017;56(5):723-31.

12. Batiha AM, AlBashtawy M. Knowledge of Philadelphia University students regarding blood donation. Transfus Med. 2013;23(3):195-8.

13. Dean BW, Hewitt SN, Begos MC, Gomez A, Messam LL. An analysis of blood donation barriers experienced by North American and Caribbean university students in Grenada, West Indies. Transfus Apheresis Sci. 2018;57(1):40-5
14. Sabu KM, Remya A, Binu VS, Vivek R. Knowledge, attitude and practice on blood donation among Health Science students in a university campus, South India. Online J Health Allied Sci. 2011;10(2):1-3.

15. Cicolini G, Comparcini D, Alfieri S, Zito E, Marta E, Tomietto M \& Simonetti V. (2019). Nursing students' knowledge and attitudes of blood donation: A multicentre study. J Clin Nurs. 2019;28 (9-10):1829-38.

16. Instituto Português do Sangue e da Transplantação. [Transfusion Activity Report and Portuguese Hemovigilance System 2017]. [Internet]. Lisbon: Instituto Português do Sangue e da Transplantação; 2017. [cited 2019 Jul 29]. Avaliable from: http://www.hemovigilancia. net/files/RA_2017_VF1.3.pdf. Portuguese.

17. Federación Española de Donantes de Sangre. [Statistical data on blood donation in Spain 2018]. [Internet]. Madrid: Federación Española de Donantes de Sangre; 2019. [cited 2019 Jul 29]. Avaliable from: http://www.hdsc.org/estadisticas-de-la-donacion-en-espana/\#2018. Spanish.

18. World Health Organization (WHO). Blood safety and availability [Internet]. Genève: WHO; 2019. [cited 2019 Ago 30]. Avaliable from: https://www.who.int/news-room/fact-sheets/detail/blood-safety-andavailability

19. Instituto Português do Sangue e da Transplantação. [Structure and Organization]. Lisbon: Instituto Português do Sangue e da Transplantação; 2017. [Internet]. [citado 2019 Jul 29]. Avaliable from: http://ipst.pt/index.php/home/estrutura-e-organizacao/centros-desangue-e-da-transplantacao. Portuguese.

20. Papagiannis D, Rachiotis G, Symvoulakis EK, Anyfantakis D, Douvlataniotis $\mathrm{K}$, Zilidis $\mathrm{C}$, et al. Blood donation knowledge and attitudes among undergraduate health science students: A crosssectional study. Transfus Apheresis Sci. 2016;54(2):303-8.

21. Martín-Santana JD \& Beerli-Palacio A. (2013). Intention of future donations: a study of donors versus non-donors. Trans Med. 2013;23(2):77-86.

22. Mohammed S, Essel HB. Motivational factors for blood donation, potential barriers, and knowledge about blood donation in first-time and repeat blood donors. BMC Hematol. 2018;18(1):36.

23. Mayaki Z, Kabo R, Moutschen M, Albert A, Dardenne N, Sondag D, et al. Knowledge, attitudes and clinical practice of blood products prescribers in Niamey. Transfus Clin Biol. 2016;23(2):78-85.

24. Gebresilase HW, Fite RO, Abeya SG. Knowledge, attitude and practice of students towards blood donation in Arsi university and Adama science and technology university: a comparative cross sectional study. BMC Hematol. 2017;17(1):20.

25. AW S. Ahmed S, Poirier J, Okeke D, Chima J, Bamisaye V \& Johnson M. Voluntary blood donation - knowledge, attitude and practice amongst students of medical schools located in the Caribbean. Afr J Microbiol Res. 2018;6(2):42-6.

26. Kanwal A, Raza AA, Saif S, Ashfaq U. Knowledge, Attitude and Practices of Voluntary Blood Donation among Students of Rawalpindi Medical University. SJRMC. 2019;23(1):50-2.

27. Henriques T, Quintal C. Young and healthy but reluctant to donate blood: an empirical study on attitudes and motivations of university students. Notas Económicas. 2018;47(47):59-73.

28. Lownik E, Riley E, Konstenius T, Riley W, McCullough J. Knowledge, attitudes and practices surveys of blood donation in developing countries. Vox Sang. 2012;103(1):64-74.

29. Alves da Silva C, Ferreira C. [Social Networks and Informal Learning of Higher Education Students]. Acción Pedagógica. 2016;25(1):6-20. Spanish. 